

\title{
Ductile damage parameters identification for cold metal forming applications
}

\author{
Pierre-Olivier Bouchard, Jean-Marie Gachet, Emile Roux
}

\section{To cite this version:}

Pierre-Olivier Bouchard, Jean-Marie Gachet, Emile Roux. Ductile damage parameters identification for cold metal forming applications. The 14th International Esaform Conference on Material Forming: ESAFORM 2011, Apr 2011, Belfast, United Kingdom. pp.47-52 - ISBN 978-0-7354-0911-8, 10.1063/1.3589490 . hal-00590272

\section{HAL Id: hal-00590272}

https://hal-mines-paristech.archives-ouvertes.fr/hal-00590272

Submitted on 9 Mar 2012

HAL is a multi-disciplinary open access archive for the deposit and dissemination of scientific research documents, whether they are published or not. The documents may come from teaching and research institutions in France or abroad, or from public or private research centers.
L'archive ouverte pluridisciplinaire HAL, est destinée au dépôt et à la diffusion de documents scientifiques de niveau recherche, publiés ou non, émanant des établissements d'enseignement et de recherche français ou étrangers, des laboratoires publics ou privés. 


\title{
Ductile damage parameters identification for cold metal forming applications
}

\author{
Pierre-Olivier Bouchard, Jean-Marie Gachet, Emile Roux \\ Mines ParisTech, CEMEF - Centre de Mise en Forme des Matériaux, CNRS UMR 7635, BP 207, 1 rue Claude Daunesse, 06904 Sophia Antipolis Cedex, France
}

\begin{abstract}
Ductile damage mechanics is essential to predict failure during cold metal forming applications. Several damage models can be found in the literature. These damage models are coupled with the mechanical behavior so as to model the progressive softening of the material due to damage growth. However, the identification of damage parameters remains an issue. In this paper, an inverse analysis approach is set-up to identify ductile damage parameters, based on different kind of mechanical tests and observables. The Lemaitre damage model is used and damage is coupled with the material behavior. The Efficient Global Optimization (EGO) method is used in a parallel environment. This global algorithm based on kriging meta-model enables the identification of a set of damage parameters based on experimental observables. Global and local observables are used to identify these parameters and a special attention is paid to the computation of the cost function. Finally, an identification procedure based on displacement field measurements is presented and applied for damage parameters identification.
\end{abstract}

Keywords: Parameters identification, inverse analysis, ductile damage, full field measurement PACS: $02.30 . Z z, 02.60 .-\mathrm{x}, 62.20 .-\mathrm{x}, 81.70 .-\mathrm{q}$

\section{INTRODUCTION}

During cold metal forming operations, the material is submitted to large plastic strain and multiaxial loadings that can potentially lead to ductile damage growth and final fracture. In order to obtain accurate and robust results, the identification of damage parameters is essential. Damage parameters identification is often performed using tensile tests load-displacement curves. This single global observable is not rich enough to identify a unique set of damage parameters. A methodology based on multiple observables is presented here. Numerical simulations are performed using the Forge ${ }^{\circledR}$ finite element software.

In a first part, the optimization algorithm developed and used in this work is presented. This algorithm is based on the Efficient Global Optimization (EGO) method [1]. This global optimization algorithm, based on kriging metamodel, is suitable for multiple minima and time-consuming problems. This algorithm is implemented in the MOOPI software (MOdular Optimization software for Parameters Identification), developed at CEMEF in the CimLib library and dedicated to optimization issues. Using MOOPI, identification can be achieved using multiple mechanical tests and the inverse analysis can be done with different finite element software.

The second part of the paper presents the classical identification methodology based on tensile tests loaddisplacement curves. Response surfaces are built for different couples of damage parameters in order to show that this single observable lead to the non-uniqueness of the solution. The definition of the cost function is an important issue, in particular when dealing with the final stage of ductile damage and fracture.

In the third part, a methodology based on full field measurements is presented. It is shown that using displacement fields enable to enrich the experimental database and to converge towards a unique solution of the identification procedure.

\section{OPTIMIZATION ALGORITHM}

To deal with the identification issue, a parallel optimization algorithm, based on the Efficient Global Optimization algorithm (EGO) suggested by Jones et al. [1], has been developed. In the MOOPI software, a parallel extension of the EGO algorithm is implemented. This algorithm is based on the construction of a meta-model of the objective function all over the parameters design space. This meta-model is built iteratively by adding one new point at each iteration. This new point is defined thanks to the maximization of the Expected Improvement (EI) criterion. 
This criterion has two goals: global minimization of the cost function and local improvement of a minimum already detected.

A parallel version of the EGO algorithm has been developed here. A flowchart of this algorithm is presented in figure 1. The main idea of this extension is the following: instead of evaluating exactly the cost function of one new set of parameters at each iteration, the idea is to temporally set the cost function value to an approximate value regarding the kriging meta-model for this new point. This temporally approximation of the cost function value is not time consuming and enables to extract a new set of parameters from the meta-model without performing a new exact evaluation (which is time consuming). $\mathrm{N}$ set of parameters can thus be extracted from the meta-model without any exact evaluation. The final step is to evaluate exactly the cost function value of these $\mathrm{N}$ new points, which can be done simultaneously using parallel computing.

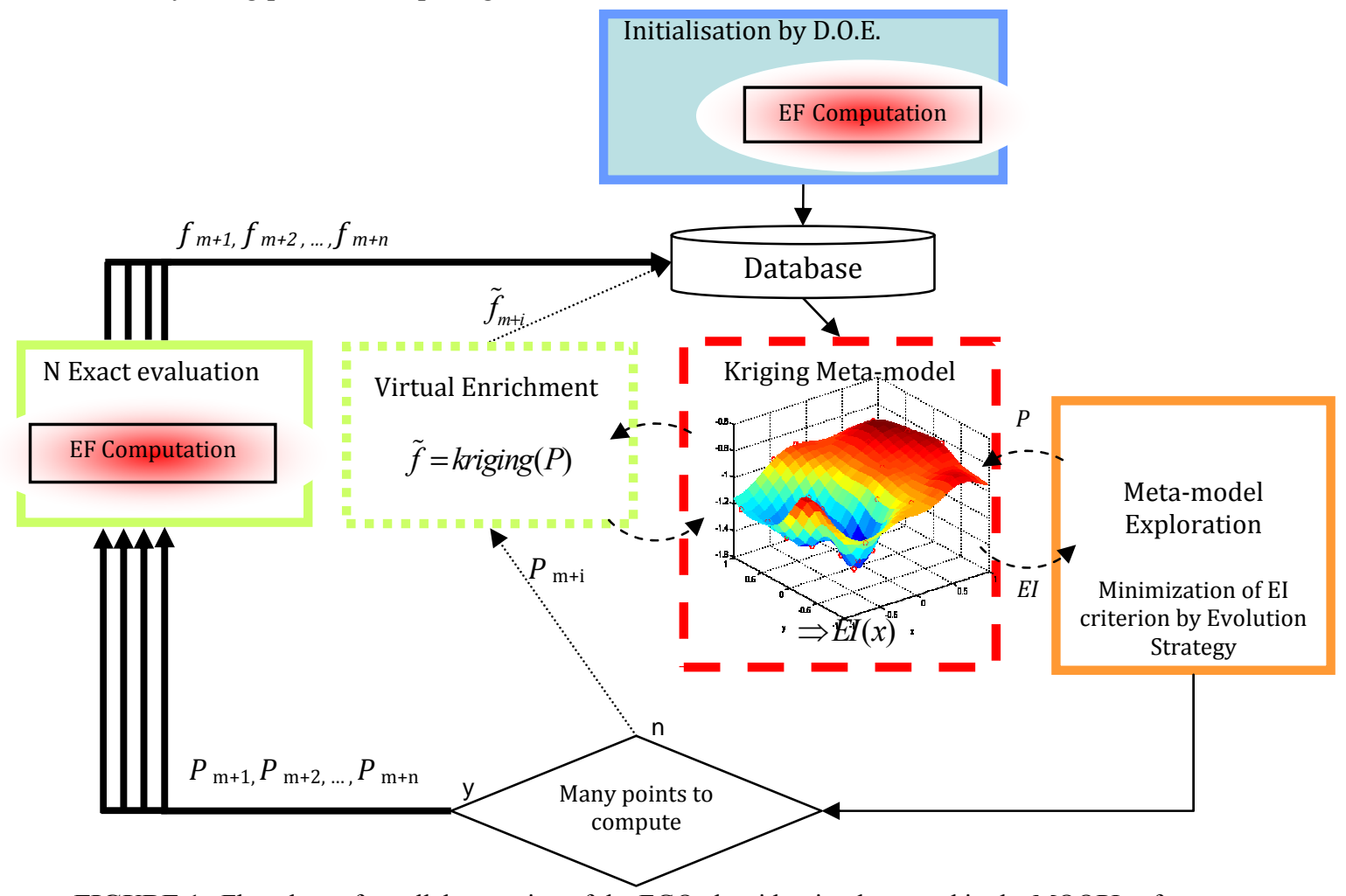

FIGURE 1. Flowchart of parallel extension of the EGO algorithm implemented in the MOOPI software

The EGO algorithm implemented in MOOPI is well suited for parameters identification by inverse analysis. This software is able to work with multiple experimental observables and multiple mechanical tests. The optimization procedure gives two main informations: the set of identified parameters and a map of the objective function all over the parameters design space. This map is particularly interesting to understand the sensitivity of the observable regarding each parameter of the model.

\section{IDENTIFICATION METHODOLOGY}

In this part, the optimization algorithm presented in the previous section is used to identify damage parameters by inverse analysis. The inverse analysis approach consists in identifying a set of material parameters by minimizing a cost function. This cost function is based on the difference between an experimental and a numerical observable. In this section, the load-displacement curve of a tensile test is the observable. When damage grows during the mechanical loading, the load-displacement curve decreases due to the progressive mechanical degradation of the material properties. This is obtained numerically by coupling damage growth with the elastoplastic mechanical behavior. In this paper, the Lemaitre damage model is used. This model will be briefly described in the first section. In a second part, it is shown that the definition of the cost function has to be carefully managed, in particular when dealing with the last stage of ductile damage and fracture. Finally, it is shown that, in some cases, such an identification can lead to multiple set of parameters that all minimize the cost function. 


\section{The Lemaitre ductile damage model}

In this paper, a modified version of the Lemaitre ductile damage model is used [2]. The Lemaitre model is a damage model coupled to plasticity based on continuum damage mechanics [3]. A scalar damage variable $D$ is defined. The material softening is obtained by replacing the equivalent stress by the effective stress (defined by equation 1) on the constitutive equations of the undamaged material.

$$
\tilde{\sigma}=\frac{\sigma}{1-D}
$$

Damage evolution is given by equations 2 and 3 where $Y$ is the strain energy release rate. $S_{0}, b$ and $\varepsilon_{\mathrm{d}}$ are material dependent parameters, $p$ is the hydrostatic pressure, $\sigma_{e q}$ is the von Mises equivalent stress.

$$
\begin{aligned}
& \left\{\begin{array}{cc}
\dot{D}=\left(\frac{Y}{S_{0}}\right)^{b} \dot{\bar{\varepsilon}}_{p l} & \text { if } \bar{\varepsilon}_{p l} \geq \varepsilon_{d} \\
\dot{D}=0 & \text { if } \bar{\varepsilon}_{p l}<\varepsilon_{d}
\end{array}\right. \\
& Y=\frac{\sigma_{e q}}{2 E(1-D)^{2}}\left(\frac{2}{3}(1+v)+3(1-2 v)\left(-\frac{p}{\sigma_{e q}}\right)^{2}\right)
\end{aligned}
$$

To model the rapid loss of load carrying capacity of the material, $D$ is set to 1 when it reaches a critical value $D_{c}$. Four parameters have to be identified $\left(S_{0}, b, \varepsilon_{\mathrm{d}}\right.$ and $\left.D_{c}\right)$.

\section{Definition of the cost function}

In this section we focus on an inverse analysis identification strategy based on conventional mechanical tests. Inverse analysis deals with the minimization of a cost function. This cost function evaluates the gap between data coming from experimental mechanical tests and from numerical simulations of the mechanical test. Formulation of the objective function for load-displacement observable is presented in equation 4. Minimization of this cost function leads to identify the hardening and damage parameters (set of parameters $P$ ).

$$
\left\{\begin{array}{l}
f \mathcal{C}_{F}(P)=\sqrt{\frac{\sum_{i=1}^{n}\left[\left(F_{i}^{\text {num }}(P)-F_{i}^{\exp }\right)\right]^{2} \Delta d_{i}}{\min \left(\sum_{i=1}^{n}\left(F_{i}^{\exp }\right)^{2} \Delta d_{i}, \sum_{i=1}^{n}\left(F_{i}^{n u m}(P)\right)^{2} \Delta d_{i}\right)}} \\
\Delta d_{i}=d_{i}-d_{i-1}
\end{array}\right.
$$

$P$ is the set of material parameters, $F^{n u m}$ and $F^{\text {exp }}$ are respectively the numerical and experimental force, $d$ the displacement, and $n$ the number of points recorded. In [4], it has been shown that particular attention has to be paid to the way the numerical and experimental breaking loads are dealt with in the computation of the cost function. In fact, if the gap between the two curves is not evaluated correctly near the breaking point, the objective function can induce wrong identified set of parameters. Our adapted strategy is illustrated in figure 2. On each curve, measurement points are added in order to complete the curve. The objective function is evaluated on the real curves completed with the additional points. This has to be done here since the decreasing part of the curve is important for the good identification of damage parameters.

This strategy allows constructing a smooth objective function, which can be efficiently used by the minimization algorithm. 


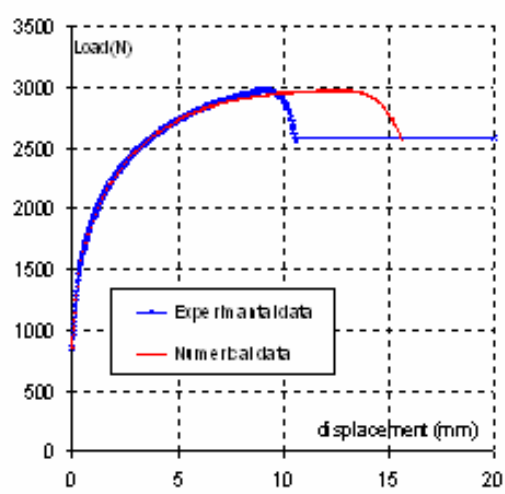

(a)

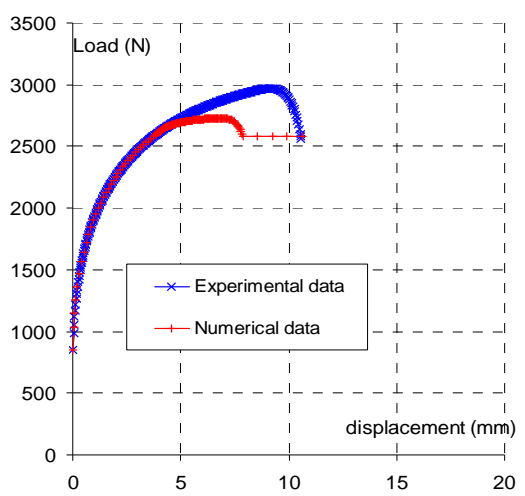

(b)

FIGURE 2. Tensile test load-displacement curves, (a) numerical fracture appears for a larger displacement value than for the experimental curve, (b) numerical fracture appears for a smaller displacement than the experimental fracture displacement

\section{Uniqueness of the solution}

When the identification of damage parameters relies on a single load-displacement curve, it can be shown that multiple set of damage parameters can reproduce accurately the experimental observable. This non-uniqueness of the inverse problem solution is illustrated in figure 3, which corresponds to the response surface of a cost function calculated on a tensile load-displacement curve for a EN AW-5774[AlMg3] aluminum alloy. This response surface exhibits two minima. Parameter $b$ is the most difficult parameter to identify. This response surface shows that the inverse problem has not only one solution with this single load-displacement observable. Therefore, it can be concluded that one single load-displacement curve is not sufficient to identify correctly a set of Lemaitre damage parameters. In order to enrich the identification basis, other experimental observables have to be considered.

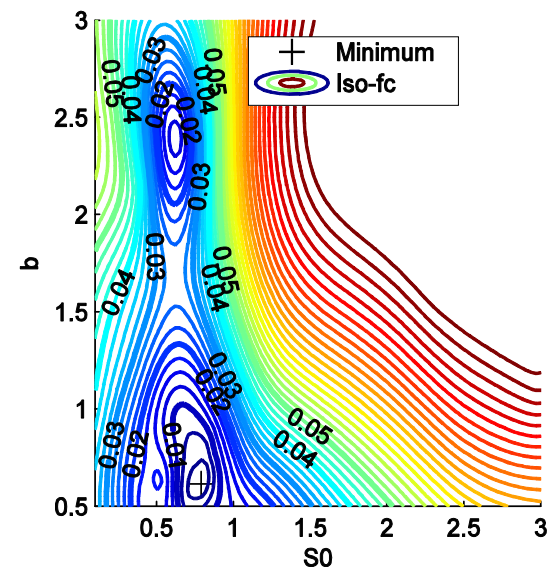

FIGURE 3. Evolution of the cost function computed with the load-displacement curve for two damage parameters $b$ and $S_{0}$ illustration of the non-uniqueness of the inverse problem (Aluminum EN AW-5774[AlMg3])

\section{IMPROVEMENT OF THE IDENTIFICATION PROCEDURE}

In order to find a single minimum of the cost function, it is necessary to enrich the experimental observables. This can be achieved using different methodologies:

- The cost function can be based on several experimental load-displacement curves (global observables) obtained using different mechanical tests: tensile tests with different specimen geometries can be used (flat, notched flat, shear induced samples ...). Such an approach is interesting for damage identification with 
different values of stress triaxiality ratio and Lode angle. It has been shown in [5] that these two parameters are essential to characterize damage growth for complex loading path.

- The cost function can be based on local observables: local displacement measurements, or full field measurements. This approach enables a better description of the local displacement fields, but requires the construction of an advanced cost function based on experimental and numerical displacement fields.

In this section, full field measurements are used for the identification. First the definition of the cost function is presented, based on displacement fields for large plastic strain. Then the methodology is applied for parameters identification purposes.

\section{Full field measurements: definition of the cost function}

The use of full field measurements for parameters identification purposes is a popular and powerful method to enrich the identification [6]. The displacement field is measured on one face of the sample by two dimensional digital image correlations (figure 4). This measurement technique allows obtaining the displacement field in the sample plan at each time of the mechanical test, till the fracture of the sample.

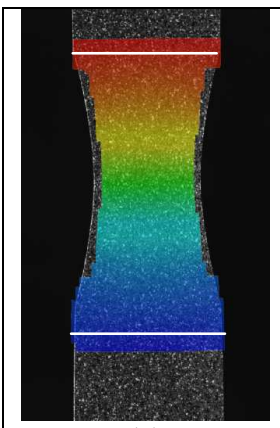

(a)

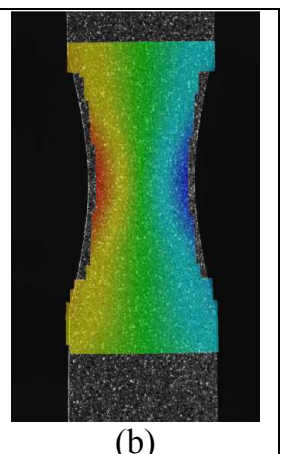

(b)

FIGURE 4. Displacement field measured by digital image correlation using the Aramis ${ }^{\circledR}$ software, (a) displacement field in the tensile direction, (b) displacement field in the transverse direction.

White lines on figure 4.a represent the border of the domain of study. Displacement fields on these two lines are use as boundary condition of the FE simulation. The aim is to reduce the size of the modeling area, in order to reduce the computation time. The use of this experimental boundary condition also eliminates any rigid body motion. This methodology can also be very efficient on more complex mechanical tests, as shear tests, to reduce the computation time.

In order to use this displacement field measurement for ductile damage parameters identification, it is important to take care of large strain. The objective function have thus to account for large displacements. The aim of the objective function is to compare at each time, the numerical displacement field $\left(U x^{n u m}\right)$ and the experimental displacement field $\left(U x^{\mathrm{exp}}\right)$. The major problem in the computation of the objective function is that, under large strain, the numerical sample shape and the experimental sample shape are different. This difference is due to the difference in terms of mechanical behavior. Consequently, the objective function has to be computed with the two fields expressed in the same configuration. At each evaluation of the objective function the two fields are thus recorded in the undeformed shape. The objective function is written as follows:

$$
f c_{x}(P)=\sum_{t=1}^{T}\left(\sqrt{\frac{\sum_{i=1}^{n}\left[\delta_{i} \cdot\left(U x_{i}^{n u m}(P)-U x_{i}^{\mathrm{exp}}\right)\right]^{2}}{\sum_{i=1}^{n} \delta_{i}}}\right)
$$


$P$ is the set of material parameters, $\delta_{i}$ is a flag value equal to one if the experimental measurement is available, zero if not, $T$ is the number of time step, and $n$ the number of measurement points.

\section{Identification based on full field measurements}

In this last section we compare the response surfaces computed with a cost function based on load-displacement curves only and with displacement field measurements (figure 4.a).

Figure 5 shows the response surfaces obtained without and with field measurements. The local minimum (red cross figure 5.a), which was already discussed in figure 3, is no more appearing on the response surface associated to displacement field measurements (figure 5.b). These two response surfaces show that using local data improves the uniqueness of the identification methodology.

a)



b)

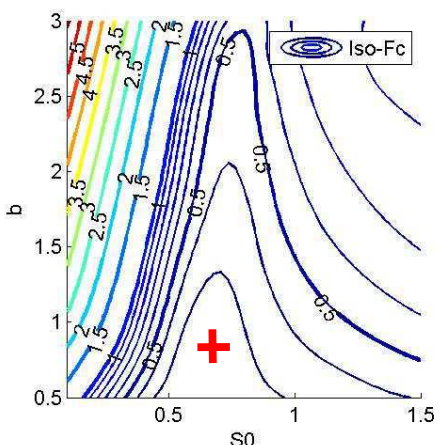

FIGURE 5. Evolution of the cost function computed with a) load-displacement curves and b) transverse displacement fields.

\section{CONCLUSION}

In this paper, different methodologies have been presented to identify hardening and ductile damage parameters using inverse analysis. The EGO algorithm has been used in a parallel environment. When load-displacement is used, special attention has to be paid to the computation of the objective function regarding the final decreasing part of the curves. It has been shown that using multiple observables is essential to improve the uniqueness and accuracy of the identification procedure. Full field measurements is undoubtedly the more appropriate solution to enrich the cost function. However, due to large plastic strain, experimental and numerical displacement fields have to be compared in the undeformed configuration.

\section{ACKNOWLEDGMENTS}

CETIM, Technical Centre for the Mechanical Industry, is gratefully acknowledged for their financial support within this study.

\section{REFERENCES}

1. D. R. Jones, M. Schonlau and W. J. Welch, Journal of global optimization, 13, 455-492 (1998).

2. P.O. Bouchard, L. Bourgeon, S. Fayolle and K. Mocellin, Int. J. Mater. Form., accepted, DOI 10.1007/s12289-010-0996-5.

3. J. Lemaitre and J.L. Chaboche, Mécanique des matériaux solides, Dunod, Paris, 2001.

4. E. Roux, M. Thonnerieux and P.O. Bouchard, Proceedings of the Tenth International Conference on Engineering Computational Technology, paper 135, 2010.

5. Y. Bao and T. Wierzbicki, International journal of Mechanical Sciences, 46, 81-98 (2004).

6. S. Avril, M. Bonnet, A.-S. Bretelle, et al., Experimental Mechanics, 48, 381-402 (2008). 